\title{
Obesity and the Factors Related to it in a High School Students Study in Tirana, Albania
}

\section{Enkelejda Shkurti}

\author{
Faculty of Technical Medical Sciences, Tirana, Albania
}

Diamant Shtiza

University Hospital Centre "Mother Theresa", Tirana, Albania

\author{
Doi:10.5901/jesr.2013.v3n7p563
}

\begin{abstract}
The aim of the study was to investigate the body weight models among adolescents school students. Overweight and obesity is a global public health concern. There is limited information on overweight and obesity in Albanian adolescents. A crosssectional study on a stratified sample of 1786 adolescents who attended school , 705( 39\%) males and 1081(61\%) females was conducted in Tirana city in 2012. They were interviewed and examined for weight and height using standardized techniques. Referring to the 2007 WHO classification for the BMI scale it resulted that $4.4 \% 95 \% \mathrm{Cl} 3.5-5.4$ adolescents resulted overweight where $32.9 \%$ were females and $67.1 \%$ were males with a statistically significant difference among them, $p<0.01$. The overall prevalence of overweight in adolescent females was $2.4 \%$, while in males was $7.5 \%$. The prevalence of obesity class I resulted $0.4 \%$, the obesity class II was $0.3 \%$ and obesity class III was $0.1 \% 95 \% \mathrm{Cl} 0.02-0.3$ from the total of participants. A remarkable finding of our study was that $70.8 \%$ of adolescent school students were in normal weight, $20 \%$ of them underweight, where $71.7 \%$ were females, $p<0.01$.
\end{abstract}

\section{Introduction}

Recently childhood and adolescent obesity has achieved epidemic ratios in developed countries (World Health Organization 2004). The high levels of adolescent overweight will have an economic consequence. According to WHO adult overweight an obesity contribute to six per cent of health expenditure in the EU (Branka F, Nikogosian H, Lobstein $T, 2002)$. Some studies propose that obesity after 3 years of age is related in the long run with a higher risk of obesity in adults, enlarged morbidity and mortality, CVD and some categories of cancer (Powers et al.1997; Freedman et al. 1999; World Health Organization,2000). There are numerous reports in the literature about childhood obesity and its undesirable effects on health from different parts of the world (World Health Organ Technical Report Series 2000; Mohammadpour-Ahranjani B, Rashidi A, Karandish M, Eshraghian MR, Kalantari N 2000-2001). During a phase of their life that is distinguished by essential psychological and physiological changes, adolescents are often attracted to unhealthy lifestyles (Reilly JJ, Armstrong J, Dorosty AR, Emmett PM, Ness A, Rogers I, Steer C, Sherriff A 2005; Yang RJ, Wang EK, Hsieh YS, Chen MY 2006) and hardly ever choose food with the best nutritional importance (Cooke LJ, Wardle J 2005; Storey KE, Forbes LE, Fraser SN, Spence JC, Plotnikoff RC, Raine KD, Hanning RM, McCargar LJ 2009). Surveillance data have indicated a high prevalence of overweight and obesity not only in developed countries but also in developing countries (Popkin BM 2001). Prevalence of overweight and obesity has increased in Albania too, a country, which has gone through fast urbanization and alterations in nutritional traditions.

Obesity estimation in childhood and adolescence would imply the hope for preventing obesity and evolution of disease which would be linked with many diseases in adulthood. The present study was designed to determine risk factors for overweight and obesity among adolescents in different regions of Albania.

\section{Methodology}

This is a cross-sectional study carried out on a national representative sample of the adolescent population in Albania aged 14-20 years from a stratified listing based on a population census available at the time of the study design. The study was conducted in January-December 2012 and the sample was composed of 1786 individuals.

The study protocol considered the invitation to fulfill a standard questionnaire that covered demographic and socioeconomic information (age, sex, place of residence, economic level) and health behaviors (cigarette smoking, alcohol 
intake, physical activity at school and during leisure time and dietary habits like food preferences) and anthropometric measurements including height, weight and the calculation of BMI. Participation was voluntary and written informed consent from the adolescent students was obtained. Measurements of body weight and height were performed by school medical doctors and students of the Faculty of Nursing in Albania, who were trained to perform this task. Height and weight were measured, using "Seca" Stadiometer (UNICEF) with beam balance, with a sensitivity of $0.1 \mathrm{~cm}$ and $0.1 \mathrm{~kg}$, respectively. Zero error was set after every 10 measurements. Height was measured without any footwear. The student stood straight with heels, buttocks, back touching the vertical limb of the instruments, and stretching upwards to the fullest extent with arms hanging on the side. The head was aligned so that the lower rim of the orbit and the auditory canal were in the horizontal plane (Frankfurt plane). Mild upward pressure was exerted on the mastoid region bilaterally. Weight was measured without any footwear with minimal clothing (school uniform).

BMI was calculated as the weight (in $\mathrm{kg}$ ) divided by the square of height( in meters). Participants in the study were divided in categories regarding their relative body weight status using WHO (WHO, 1997) criteria (underweight BMI < 18,5 kg/m², normal: BMI= 18,5-24,9 kg/m², overweight: $\mathrm{BMI}=25,0-29,9 \mathrm{~kg} / \mathrm{m}^{2}$, obese : $\mathrm{BMI} \geq 30,0 \mathrm{~kg} / \mathrm{m}^{2}$ )

Estimation of the prevalence of overweight and obesity was based on the cut-off points of the International Obesity Task Force (IOTF) standards (Cole TJ, Bellizzi MC, Flegal KM, Dietz WH 2000). In addition to the 2007 WHO reference, prevalence data were calculated using the widely used 2000 Center for Disease Control (CDC) reference for comparison. The dietary history was assessed by asking the students what meals did they consume regularly, if they took their food in a fast-food two or more times in a week, what kind of cereals, vegetables, fruits or juices did they have last week etc. While the physical activity was estimated by asking the students about the frequency of being physically active for a total of 60 minutes in a day, and the average of the outdoor games(dancing, football, volleyball, basketball), while the sedentary lifestyle was evaluated by asking the duration of watching television and using computers and internet. The psychological perception of weight was appraised by questions related to the self-definition of weight, dieting and the actions taken regarding the subject's weight. The statistical analyses were performed with SPSS 15.0 software for Windows (SPSS Inc., Chicago, IL, USA). For each survey, means and standard deviations were calculated for each sex. Mean values were compared using the Student's t test. To define overweight and obesity based on BMI, observed and expected numbers in each category were compared using the $x^{2}$ test. Pearson's correlation analysis was used to reveal the relationships between obesity and age groups. It was decided to use a conservative statistical significance of $\mathrm{P}=0.01$ to reduce the Type 1 error.

\section{Results}

The histogram (figure.1) is related to normal distribution. It is perceived a domination of the group age 14-17 years old. The median of the participants in the study is 16 years old.

Figure 1. Histogram of the age of the participants in the study.

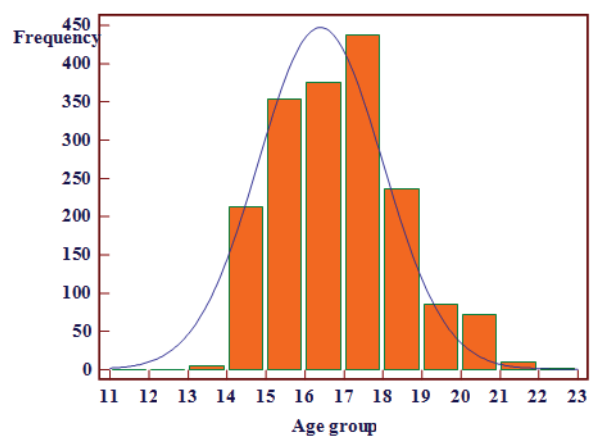

79 (4.4\%) students result in severe underweight considering the classification of BMI scale , 63 (79.7\%) from them were females and $16(20.3 \%)$ males with a statistically significant difference among them, $p<0.01$

$357(20 \%)$ of the participants were underweight $256(71.7 \%)$ from which were females and 101 (28.3\%) males with a statistically significant difference among them, $p<0.01$. 
It is noticed a predominance of the normal weight with 1264 (20\%) of the participants, with a statistically significant difference with the other categories of weight $\left(X^{2}=66.8 \quad p<0.01\right) .735(58.1 \%)$ students were females and $529(41.9 \%)$ males where $p<0.01$.

$79(4.4 \% 95 \% \mathrm{Cl} 3.5-5.4)$ adolescents were overweight where 26 (32.9\%) were females and 53 (67.1\%) males with a statistically significant difference between them, $p<0.01$.

Prevalence of overweight in females was 2.4\% (26/1081) while in males was 7.5\% (53/705).

It is observed a majority of overweight in males compared to females where $p<0.01$ (Table.1).

Table 1. Classification of the students prior to BMI scale and gender

\begin{tabular}{lccc} 
BMI scale & Females & Males & Total \\
Severe underweight & 63 & 16 & $79(4.4 \%)$ \\
Underweight & 256 & 101 & $357(20.0 \%)$ \\
Normal & 735 & 529 & $1264(70.8 \%)$ \\
Overweight & 26 & 53 & $79(4.4 \%)$ \\
Obese Grade I & 0 & 3 & $3(0.2 \%)$ \\
Obese Grade II & 0 & 2 & $2(0.1 \%)$ \\
Obese Grade III & 1 & 1 & $2(0.1 \%)$ \\
\hline Total & 1081 & 705 & 1786
\end{tabular}

It is detected a weak positive correlation, but statistically important between BMI and age of the participants in the study. As the age of students increases, the level of BMI grows up too $(r=0.18 p<0.01)($ Table.2)

Table 2. Correlation of BMI with Age

$\begin{array}{ll}\text { Sample size } & 1786 \\ \text { The Pearson coefficient of correlation } \mathbf{r} & 0.1835 \\ \text { Significance level } & \mathbf{P}<0.0001 \\ 95 \% \text { Interval of confidence for } \mathbf{r} & 0.1383-0.2279\end{array}$

We identified that 4.3\% (9/210) of the participants with high economic level, 4.5\% (67/1496) of the students with medium economic level and $3.8 \%$ (3/80) of them with low economic level were overweight. It is not detected an important significance difference between the economic level and overweight $\left(X^{2}=3.3, p=0.1\right)(T a b l e .3)$

Table 3. Classification of the students concerning BMI scale and the economic level

\begin{tabular}{lcccc} 
BMI scale & High & $\begin{array}{c}\text { Economic level } \\
\text { Medium }\end{array}$ & Low & Total \\
\hline Severely underweight & 11 & 63 & 5 & $79(4.4 \%)$ \\
Underweight & 50 & 295 & 12 & $357(20.0 \%)$ \\
Normal & 140 & 1065 & 59 & $1264(70.8 \%)$ \\
Overweight & 9 & 67 & 3 & $79(4.4 \%)$ \\
Obese Grade I & 0 & 3 & 0 & $3(0.2 \%)$ \\
Obese Grade II & 0 & 1 & 1 & $2(0.1 \%)$ \\
Obese Grade III & 0 & 2 & 0 & $2(0.1 \%)$ \\
\hline Total & $\mathbf{2 1 0}$ & $\mathbf{1 4 9 6}$ & $\mathbf{8 0}$ & 1786
\end{tabular}

It is noted a weak positive correlation but statistically important between BMI and smoking of the adolescents. increase BMI values increase in the participants that are regular smokers and ex-smokers $(r=0.127 p<0.01)($ Table.4)

Table 4. Correlation of BMI with smoking

$\begin{array}{ll}\text { Sample size } & 1786 \\ \text { The coefficient of correlation of the ranks of Spearman (rho) } & 0.127 \\ \text { Level of significance } & P<0.0001 \\ 95 \% \text { Interval of Confidence for rho } & 0.083-0.1\end{array}$


The majority of students in normal weight, 1011or 80\% (1011/1264) of them have a right perception of their weight with a statistically significant difference with the other categories of perception $\left(x^{2}=2906.1 \quad p<0.01\right) .43(54.4 \%)$ of the participants have a proper perception that are "slightly overweight" with a statistically significant difference with the other categories of perception $\left(X^{2}=61.2 p<0.01\right)($ Table.5)

Table 5. Comparison of BMI scale and the psychological perception of the weight from the subjects

\begin{tabular}{ccccccc} 
BMI scale & "nearly in the & $\begin{array}{c}\text { Psychological weight perception } \\
\text { "slightly }\end{array}$ & $\begin{array}{c}\text { "slightly } \\
\text { "considerably } \\
\text { proper weight" } \\
\text { overweight" }\end{array}$ & $\begin{array}{c}\text { "considerably } \\
\text { underweight" }\end{array}$ & Total \\
\hline $\begin{array}{c}\text { Severely } \\
\text { underweight }\end{array}$ & 52 & 1 & 21 & 0 & 5 & $79(4.4 \%)$ \\
Underweight & 269 & 7 & 73 & 2 & 6 & $357(20.0 \%)$ \\
Normal & 1011 & 160 & 80 & 9 & 4 & $1264(70.8 \%)$ \\
Overweight & 30 & 43 & 4 & 2 & 0 & $79(4.4 \%)$ \\
Obese G. & 1 & 2 & 0 & 0 & 0 & $3(0.2 \%)$ \\
Obese G. II & 1 & 0 & 0 & 1 & 0 & $2(0.1 \%)$ \\
Obese G.III & 2 & 0 & 0 & 0 & 0 & $2(0.1 \%)$ \\
\hline
\end{tabular}

\section{Discussion}

Despite economic, social, cultural and religious disparities among them, overweight and obesity are turned into a widespread matter common for all uncommonly distant countries.

Regarding the data provided from a previous study conducted in Tirana, the capital of Albania in 2001, about body weight patterns it was found that the prevalence of obesity in men was 22\% and 30\% in women (Laidon Shapo, Joceline Pomerlea, Martin McKee, Richard Coke and Agron Ylli 2001).

Concerning our study we have noticed that there was quite a big change in the levels of overweight and obesity in 2001 that were very high in the adult population, while in the adolescent school students they were very low (4.4\%). The increase in the levels of prevalence of overweight and obesity after the communist regime till the year 2000, was dedicated to the economic and cultural changes after the communist regime and the establishment of democracy and the opening of borders in Albania that has followed a free market economy as well as in the media influence and the nutritional changes mainly a growing fast food availability. People in big cities such as Tirana have experienced an evident progress in the standard of living and have become attracted to a more consumptive lifestyle which may lead to the rising prevalence of overweight and obesity. Based on the data on other countries in transition (Pudule I, Grinberga D, Kadziauskiene K, Abaravicius A,Vaask S, Robertson A, et al 1999; Pomerleau J, McKee M, Robertson A, Vaask S, Kadziauskiene $\mathrm{K}$, Abaravicius $\mathrm{A}$, et al 2000), it has been possible that the increased urbanization and the modernization of lifestyle (dietary changes, increased inactivity, smoking) have played a key role in this change.

While nowadays, regarding our study we have to say that the levels of overweight and obesity concerning adolescents in Albania (4.4\%; $0.4 \%$ ) are among the lowest in Europe, regarding the data from IOTF international cut off points for global trends in obesity. So we may compare our data on overweight with the data of Latvia, Estonia and Turkey.

There has been a wide range of prevalence of overweight and obesity worldwide, ranging from India, where only $0.7 \%$ of the population is obese, to French Polynesia, where nearly $40 \%$ of the population is obese ( Nguyen DM, ElSerag HB 2009). In countries such as China and Thailand (Wang Y, Wang JQ 2002; Zhou H, Yamauchi T, Natsuhara K, Yan Z, Lin H, Ichimaru N, et al, Kim SW, Ishii M, Ohtsuka R 2006), the incidence of overweight and obesity is more frequently observed in boys than in girls, whereas in the United Arab Emirates there are more girls diagnosed as overweight and obese (Malik M, Bakir A 2007). It is the same in Albania where the prevalence of overweight and obesity is higher in boys than in girls.

Regarding the data provided from IOFT cut off points for Albania in 2008, the prevalence of obesity for males was 8.5\% and for females was $9.7 \%$ (International Association for the Study of Obesity 2012). So, there was a big difference in the prevalence of overweight between the adolescent and the adult population in Albania. The high obesity prevalence noticed along with the recent decrease in physical activity, dietary changes and increase in smoking prevalence has led to considerable increases in many non-communicable diseases in Albania in the coming decades. Health promotion strategies are needed that prevent excess weight gain in the Albanian adult population. One of the strengths of this study 
would be that is has been anonymous and based on a large nationally representative sample. Among the limitations would be the cross-section nature of the survey preventing any conclusions regarding causality and the non-inclusion of adolescents who have dropped out of school and who might be more vulnerable. As a result further research is needed to include in the study also the adolescents who did not attend school.

\section{Conclusions}

An argument related to these low levels of adolescent overweight and obesity in Albania would be the Mediterranean diet with plenty of fruits and vegetables, the meals consumed at home or more healthy foods, the increase of physical activity, gym, aerobics, the tendency of boys and girls to take care about their image, influenced by the media and TV that promote the slim and elegant individuals as a way to be successful in life. The more recent increase in social pressure on males setting somewhat an unrealistic body ideal consisting of a low body fat and a high muscularity figure could lead to a considerable increase in disordered eating (McCabe MP, Ricciardelli LA 2004). Actually, body and weight concerns are universally considered as typical female concerns. So, the social pressure to be thin has a greater influence on girls.

An interesting finding in our study was that a high percentage of adolescent school students almost $70.8 \%$ were in normal weight. Identifying pathways would seem essential for the design of programs in the field of adolescent health promotion and positive health. In our opinion, the low levels of overweight and obesity and the huge proportion of normal weight within the adolescent population was dedicated to a popularity of healthy lifestyles among the youngsters.

However, another exciting conclusion of our study was that $20 \%$ of the participants, mostly girls reflected underweight problems. This is a serious issue, as a consequence strategies should be implemented to tackle this problem in Albania, in the near future.

\section{References}

Branka F, Nikogosian H, Lobstein T, eds. The challlenge of obesity in the WHO

European Region and the strategies for respons. Copenhagen: WHO 2002.

Body weight patterns in a country in transition: a population-based survey in Tirana City, Albania Laidon Shapo1, ${ }^{*}$, Joceline Pomerleau1, Martin McKee1, Richard Coker1 and Agron Ylli 2 1European Centre on Health of Societies in Transition, London School of Hygiene and Tropical Medicine, Keppel Street, London WC1E 7HT, UK: 2Endocrinology Department, University Hospital Centre 'Mother Theresa', Dibra Street, 370 Tirana, Albania

Cooke LJ, Wardle J. Age and gender differences in children's food preferences. Br J Nutr. 2005;93:741-746. doi: 10.1079/BJN20051389

Cole TJ, Bellizzi MC, Flegal KM, Dietz WH. Establishing a standard definition for child overweight and obesity worldwide: international survey. BMJ. 2000;320:1-6.

International Association for the Study of Obesity. London-January 2012

Malik M, Bakir A (2007) Prevalence of overweight and obesity among children in the United Arab Emirates. Obes Rev 8(1):15-20 .

McCabe MP, Ricciardelli LA. A longitudinal study of pubertal timing and extreme body change behaviors among adolescent boys and girls. Adolescence 2004;39:145-166.

Mohammadpour-Ahranjani B, Rashidi A, Karandish M, Eshraghian MR, Kalantari N. Prevalence of overweight and obesity in adolescent Tehrani students, 2000-2001: an epidemic health problem. Public Health Nutr. 2004;7:645-648.

Nguyen DM, El-Serag HB. The big burden of obesity. Gastrointest Endosc. 2009;70:752-757. Germany.

Pomerleau J, McKee M, Robertson A, Vaask S, Kadziauskiene K, Abaravicius A, et al. Physical inactivity in the Baltic countries. Preventive Medicine 2000; 31: 665-672.

Popkin BM. The nutrition transition and obesity in the developing world. J Nutr. 2001;131:871S-873S.

Powers et al.1997; Freedman et al. 1999; World Health Organization,2000).

Pudule I, Grinberga D, Kadziauskiene K, Abaravicius A,Vaask S, Robertson A, et al. Patterns of smoking in the Baltic Republics. Journal of Epidemiology and Community Health 1999; 53: 277-28

Reilly JJ, Armstrong J, Dorosty AR, Emmett PM, Ness A, Rogers I, Steer C, Sherriff A. Early life risk factors for obesity in childhood: cohort study. Bmj. 2005;330:1357. doi: 10.1136/bmj.38470.670903.E0.

Storey KE, Forbes LE, Fraser SN, Spence JC, Plotnikoff RC, Raine KD, Hanning RM, McCargar LJ. Diet quality, nutrition and physical activity among adolescents: the Web-SPAN (Web-Survey of Physical Activity and Nutrition) project. Public Health Nutr. 2009;12:2009-2017. doi: 10.1017/S1368980009990292.

Wang Y, Wang JQ (2002) A comparison of international references for the assessment of child and adolescent overweight and obesity in different populations. Am J Clin Nutr 56:973-982

World Health Organization (2004) World Health Assembly resolution WHA57.17 On a global strategy on diet physical activity and health. Geneva: WHO.

World Health Organ Technical Report Series. Obesity: preventing and managing the global epidemic. Report of a WHO consultation. 
World Health Organ Tech Rep Ser. 2000;894:1-253.

World Health Organization (WHO). Obesity. Preventing and managing the Global Epidemic. Geneva: WHO, 1997.

Yang RJ, Wang EK, Hsieh YS, Chen MY. Irregular breakfast eating and health status among adolescents in Taiwan. BMC Public Health. 2006;6:295. doi: 10.1186/1471-2458-6-295.

Zhou H, Yamauchi T, Natsuhara K, Yan Z, Lin H, Ichimaru N, et al, Kim SW, Ishii M, Ohtsuka R (2006) Overweight in urban schoolchildren assessed by body mass index and body fat mass in Dalian, China. J Physiol Anthropol 25(1):41-48 